\title{
Universal Measure for Medical Image Quality Evaluation Based on Gradient Approach
}

\author{
Marzena Bielecka ${ }^{1(\bowtie)}$, Andrzej Bielecki², Rafał Obuchowicz ${ }^{3}$, \\ and Adam Piórkowski ${ }^{4}$
}

1 Faculty of Geology, Geophysics and Environmental Protection, Chair of Geoinformatics and Applied Computer Science, AGH University of Science and Technology, Mickiewicza Ave. 30, 30-059 Cracow, Poland bielecka@agh.edu.pl

${ }^{2}$ Faculty of Electrical Engineering, Automation, Computer Science and Biomedical Engineering, Chair of Applied Computer Science, AGH University of Science and Technology, Mickiewicza Ave. 30, 30-059 Cracow, Poland bielecki@agh.edu.pl, azbielecki@gmail.com

3 Department of Diagnostic Imaging, Jagiellonian University Medical College, Kopernika Street 19, 31-501 Cracow, Poland r.obuchowicz@gmail.com

4 Faculty of Electrical Engineering, Automation, Computer Science and Biomedical Engineering, Chair of Biocybernetics and Biomedical Engineering, AGH University of Science and Technology, Mickiewicza Ave. 30, 30-059 Cracow, Poland pioro@agh.edu.pl

\begin{abstract}
In this paper, a new universal measure of medical images quality is proposed. The measure is based on the analysis of the image by using gradient methods. The number of isolated peaks in the examined image, as a function of the threshold value, is the basis of the assessment of the image quality. It turns out that for higher quality images the curvature of the graph of the said function has a higher value for lower threshold values. On the basis of the observed property, a new method of no-reference image quality assessment has been created. The experimental verification confirmed the method efficiency. The correlation between the arrangement depending on the image quality done by an expert and by using the proposed method is equal to 0.74 . This means that the proposed method gives a correlation of higher than the best methods described in the literature. The proposed measure is useful to maximize the image quality while minimizing the time of medical examination.
\end{abstract}

Keywords: Medical imaging · Imaging quality assessment $\cdot$ Gradient methods

\section{Introduction}

Computer imaging is one of the crucial problems in contemporary computer science. Medical imaging is strictly connected to this topic. Medical images obtained

(C) Springer Nature Switzerland AG 2020

V. V. Krzhizhanovskaya et al. (Eds.): ICCS 2020, LNCS 12140, pp. 406-417, 2020.

https://doi.org/10.1007/978-3-030-50423-6_30 
by using various techniques - see Sect. 3 - are encoded in digital form. Since the images are analyzed by experts for diagnostic purposes, good image quality is necessary to detect all pathological changes, especially in the context of finding lesions at an early stage. On the other hand, a great number of medical images is the reason a great demand of their computer analysis. Various methods of artificial intelligence (see [12]) are widely used for this purpose. Good image quality is essential for the analyzing algorithm to work effectively. It is particularly important when syntactic methods are used, which are characterized by high sensitivity to any disturbances occurring in the analyzed image [13]. Syntactic methods are often used to analyze medical images [1-5,25-27]. Therefore it is important to get high quality images. However, in order to obtain a good quality image, a sufficiently long examination time is necessary, which in some cases, e.g. X-ray images, involves the patient's exposure to harmful radiation. Therefore, it is important to develop methods that will maximize image quality while minimizing time of medical examination. Obtaining a precise measure of image quality, which is the subject of this paper, is one of the issues in this undertaking.

\section{Medical Imaging Quality in Computer Science}

Image quality assessment is an important field not only in the IT or entertainment industry, but also in medicine. Based on the control of image parameters, the radiation dose or acquisition time is reduced. Unfortunately, standard parameters such as Signal-to-Noise Ratio (SNR) or Contrast-to-Noise Ratio (CNR) do not work in this area. Examples can be given where, despite the high parameters, the images have difficult or doubtful diagnostic value. For this reason, the search for new assessment methods is still a challenge [6].

Image quality assessment methods can be divided into methods based on reference images (Partial or Full Reference-Image Quality Assessment) and methods without reference (No Reference - Image Quality Assurance, NR-IQA) [7,33]. In the first case these are well-known measures such as SNR [10], PSNR (Peak Signal-to-Noise-Ratio) [17], Contrast-to-NoiseRatio (CNR) [15], and Structural Similarity Index (SSIM) [16]. These measurements require a reference to humanpointed images as reference. The second group (NR-IQA) are methods that specify a blind measure for images without referring to the indicated quality sample $[8,28,30,31]$. In this case, it is easier to compare the quality of images for different modalities, although these measures do not correlate so well with the subjective assessments of people.

No-reference measures have a different construction. For example, a measure, based on structural MRI and two types of analysis of variance, was proposed in [34]. An attempt, based on applying multidirectional filters to MR images and then examining the feature statistics, is described in [19]. In [29] the authors applied Bayes theory to the relationship between entropy and image quality attributes. Although the subject of pulse noise removal in magnetic resonance imaging is well known [21], no one has ever tried to evaluate the quality of this imaging by quantifying this noise. This issue is the subject of this work. 


\section{Medical Imaging Quality in Clinical Practice}

To obtain best imaging quality in the techniques where $\mathrm{X}$-rays are used both in conventional radiography (CR) and the computed tomography (CT) but also where RF beam is used - as in magnetic resonance (MR) - one has to meet two main criteria. First is to produce possibly best - hence diagnostic image, and second - to provide an examination in a way most safe and comfortable for the patient as it is possible. In techniques where X-rays are used the criterion of image quality is met where the contrast of structures with different absorption of X-rays represented on the image is sufficiently different and imaged structures can be recognized as separate elements. Reduction of the tube voltage $(\mathrm{kV})$ and, as a consequence, the reduction of X-rays energy provides good differentiation of the imaged objects. On the other hand, higher energy of X-rays enables to reduce dose absorbed by the human body. Therefore images with higher $\mathrm{kV}$ are preferred. Image quality in radiography is also strongly affected by the noise (mottle) which may decrease quality of the imaged object borders and hence limits its proper recognition [18]. Both in CR and CT the mottle is usually caused by a decrease of photons quantity used to create the image and typically associated with a reduction of X-ray tube current - amperage (mAs). Although its increase will improve image contrast, one has to remember that the increase of the amperage will result in a proportional increase in the radiation dose. Therefore, good quality of the radiographic pictures comes as an effect of the interplay between radiation dose and diagnostic performance of the image, built on proper contrast to noise ratio. Choice of appropriate parameters for sufficient contrast of the image and acceptable noise should be influenced by the clinical situation (body region and structures needed to be visualized). Beam filtration medium is another important physical determinant of X-ray beam quality usually preselected to the specific imaging tasks [11]. Similar principles of the image creation are actual for the CT due to helical movement of the X-ray tube around the patient body. One must remember that scan time, proportional to radiation dose, is longer. Slice thickness also affects radiation dose entering each detector but also patients body. Currently most commonly used CTDI (CT dose index) is a parameter that describes the dose output of a scanner [20]. It should be stressed that spacing between slices is often equal to the slice thickness - there is a close relationship between consecutive slices that are practically continuous. Therefore one describes cumulative dose from slice series as multiple-slice average dose (MSAD) and describes 1.25 to 1,4 higher dose than in single slice dose. Cone beam CT (CBCT) is free of the aforementioned phenomena, as there is no helical movement of the X-ray tube. Therefore, cone shape X-ray beam is used and average dose can to ten times (in comparison to the helical scan of the head) but information according to soft tissue cannot be represented in diagnostically usable form what is the major drawback of the CBCT. As CBCT allows to a substantial reduction of the dose still mean radiation dose in CBCT exceeds over three times that in $\mathrm{CR}$ technique [32]. In magnetic resonance technique radiation is not used and technique is reported as harmless to the human body. The only consideration is scanning time - substantially longer than in CT technique 
what combined with high susceptibility to the motion artefacts makes MR useless for unconscious patients or persons unable to withstand longer in the still position due to pain or altered posture. Therefore in MR quality of an image is a tradeoff time and picture characteristics. There are a plethora of parameters that might influence image quality. From geometric features of the MR image, the increase of matrix size will decrease signal although image produced will be sharper due to the decrease of pixel volume. To overcome signal drop mostly averages increase is used however this is in the expense of a substantial increase of scanning time. At the opposite, one can put the field of view (FOV) changes - if the increased amount of signal is also increased what comes with pixel size multiplication (with the image sharpness reduction). On similar principle works another geometrical parameter of MR image - a slice size - can be changed. Amount of signal will be increased by slice size as more signal is captured in the bigger voxel. Clinical usefulness of such image, however, is not always provided. Sequence parameters as TR and TE have to be manipulated with caution as those parameters are strictly valued for appropriate sequences [14]. Currently, image quality in MR is estimated parametrically by comparison of the image noise to the background noise what is expressed as SNR and this value is displayed on the operator console. This parameter, however, not always expresses subjective assumption of the image and it is useful for the mathematical estimation of the presumptive image signal loss [22]. This assumption is particularly useful during sequence planning, where time reduction is desired because of the clinical situation. Awareness of the amount of signal perceived from the planned sequence is needed especially where useful tools as parallel imaging are used. This technique called Grappa by Simens vendor allows for substantial reduction of exam time on the expanse of undersampled signal [9]. Amount of the signal and time of examination in the case of MR but also dose quantity and image quality in $\mathrm{CR}$ and $\mathrm{CT}$ is a topic of continuous discussion and research. It is also a field of technological improvements. Therefore, objective and repetitive measures of the image quality are awaited by the medical community as tools indispensable to provide patient safety and comfort.

\section{Impulse Noise Quantification}

Image quality assessment is often subjective. The authors noticed that there is some correlation between inferior image quality ratings and the impulse noise present on it. Therefore, they decided to try to quantify this relationship.

The proposed method is based on pixel counting, which predominates brightness over its surroundings (4-connected neighborhood) by a given threshold $t$ or more - see Formula (1). Two directions are considered: the selected points are lighter than their surroundings or darker than their surroundings. For a given image, it is possible to assess how this number changes with the increase of the threshold. To keep the reference level, all images are scaled to a range of 8 bits $(0-255)$. 


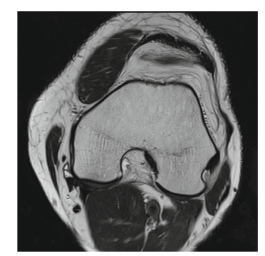

(a) input, Q1

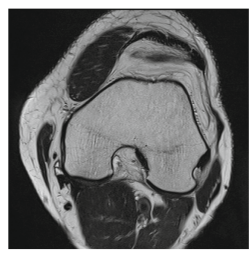

(e) input, Q2

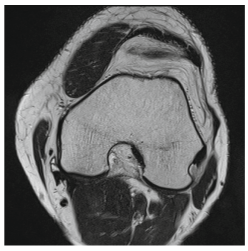

(i) input, Q3

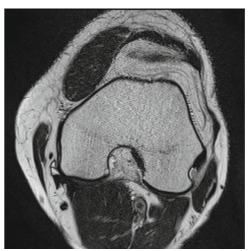

(m) input, Q4

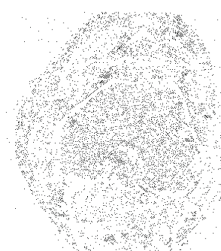

(b) $t=1$

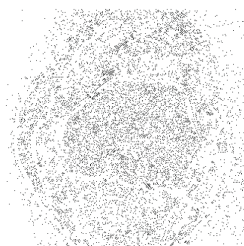

(f) $t=1$

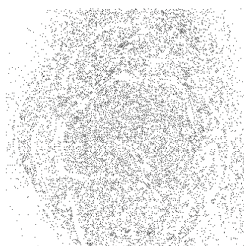

(j) $t=1$

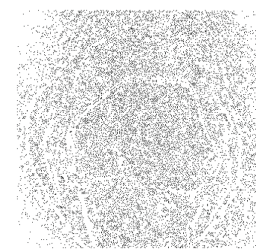

(n) $\mathrm{t}=1$ (c) $\mathrm{t}=5$

(d) $\mathrm{t}=10$ (g) $t=5$

(h) $\mathrm{t}=10$ (k) $\mathrm{t}=5$

(l) $\mathrm{t}=10$

Fig. 1. Images with gradual reduction of acquisition time and decreasing quality.

Projections of the same patient, acquired by using magnetic resonance imaging, are shown in Fig. 1. Four successively reduced acquisition times, which resulted in a gradual decrease in quality, are presented - see also [24]. Pixels that dominated or that were dominated by the surroundings by level at least of 1,5 and 10 were determined for these images, and they are presented in the following columns in the figure. It can be seen that as the set threshold increases, their number decreases, which is precisely presented in the plot - see Fig. 2 and Fig. 3. It should be noted that for better quality images, the number of indicated points for the same threshold is smaller than for images of lower quality - this process is gradual. The analysis of the presented relationship is the basis for determining the quality of the image. 
Let us define the following logical conditions that describe whether the grey level $P(x, y)$ of the pixel $(x, y)$ in the pixel matrix $M \times N$ differs from the grey level of neighboring pixels vertically and horizontally, respectively, by more than the threshold value $t$ :

$$
\begin{aligned}
& \phi_{\text {ver }}^{+}(x, y):(P(x, y)>P(x, y+1)+t) \wedge(P(x, y)>P(x, y-1)+t), \\
& \phi_{\text {hor }}^{+}(x, y):(P(x, y)>P(x+1, y)+t) \wedge(P(x, y)>P(x-1, y)+t), \\
& \phi_{\text {ver }}^{-}(x, y):(P(x, y)>P(x, y+1)-t) \wedge(P(x, y)>P(x, y-1)-t), \\
& \phi_{\text {hor }}^{-}(x, y):(P(x, y)>P(x+1, y)-t) \wedge(P(x, y)>P(x-1, y)-t),
\end{aligned}
$$

where $x \in\{1, \ldots, M-1\}, y \in\{1, \ldots, N-1\}$. The below logical condition described by the propositional function $\phi(x, y)$ is satisfied if the grey level of the pixel $(x, y)$ differs from the grey level of neighboring pixels by more than the threshold value $t$ :

$$
\phi(x, y)=\left(\phi_{y}^{+}(x, y) \wedge \phi_{x}^{+}(x, y)\right) \vee\left(\phi_{y}^{-}(x, y) \wedge \phi_{x}^{-}(x, y)\right)
$$

Let $A(t)$ be the set of the pixels that satisfy the specified condition (1), i.e. $A(t)=\{(x, y): \phi(x, y)\}$ and let $I(t)$ denotes the number of such pixels, i.e. $I(t)=\# A(t)$. The study of some geometrical properties of the graph of the function $I(t)$ is the basis of the proposed method - see the next section.

\section{Description of the Method}

Gradient methods allow the researcher to analyze the local properties of the functions $F: \mathbb{R}^{n} \rightarrow \mathbb{R}$ and, as a consequence, the local properties of the graphs of the functions, i.e. the sets of the following form $\left\{\left(x_{1}, \ldots, x_{n}, f\left(x_{1}, \ldots, x_{n}\right)\right)\right\}$. If $n=2$, then the graph of the function has natural interpretation in the context of computer graphics. In particular, let us assume that $\mathbb{R}^{2}$ is discretized and $f\left(x_{1}, x_{2}\right)$ is defined on a finite rectangle - the pixel matrix - that is a subset of the discretized $\mathbb{R}^{2}$. If, furthermore, the values of the function $f$ are values of gray level of individual pixels, then gradient methods can be used to analysis and processing of the digital image. In this paper such approach is applied to medical images.

In this sort of images, individual areas in the image correspond to individual tissues. Therefore, in good quality images, in the mentioned areas, the gray level should be more or less constant, whereas the boundaries between areas should be clear - see Fig. 2. As a consequence, the number of isolated peaks $I(t)$ (see Sect.4), as a function of the threshold value $t$, in good quality images should decrease rapidly and then flatten - see Fig. 3. Thus, the value of the maximum curvature of the graph of this function and the point in which it is reached provide important information about the quality of the image. 


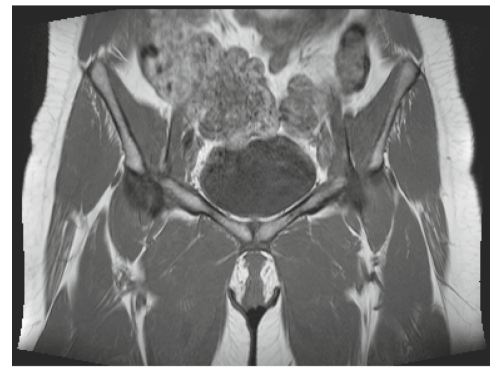

(a)

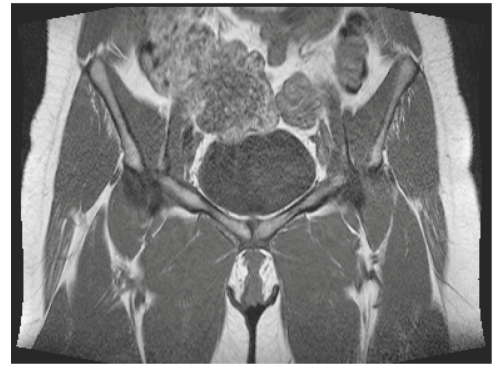

(c)

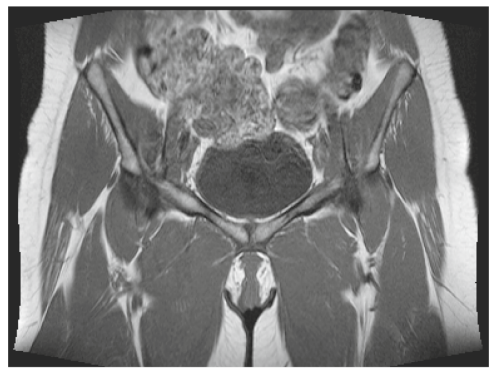

(b)

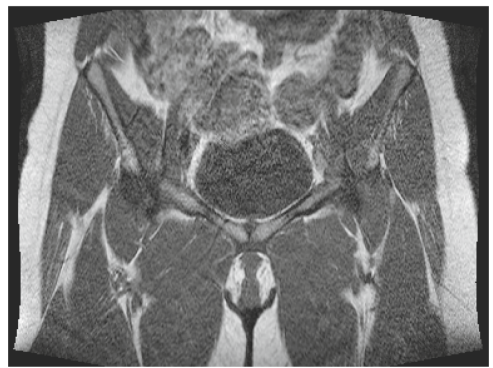

(d)

Fig. 2. Four images of the pelvis of the same patient P01. The images are sorted according to decreasing quality. Thus, image (a) is the best one, whereas image (d) is the worst one.

In the proposed method, the curve was fitted to the points obtained in this way. A few fitting functions were tested and it turned out that for the following formula

$$
f(x)=a \cdot x^{b},
$$

where $a$ and $b$ are the fitted parameters, the goodness of fit $R^{2}$ is close to 1 . The curvature $\kappa$ at the point $\left(x_{0}, y_{0}\right)$ of the curve described by a function $y=f(x)$ is given by the formula

$$
\kappa\left(x_{0}, y_{0}\right)=\frac{\left|y_{0}^{\prime \prime}\right|}{\sqrt{\left(1+y_{0}^{\prime}\right)^{3}}}
$$

For each curve its maximum curvature has been calculated numerically. The point, in which this maximum is realized, has been detected as well. 


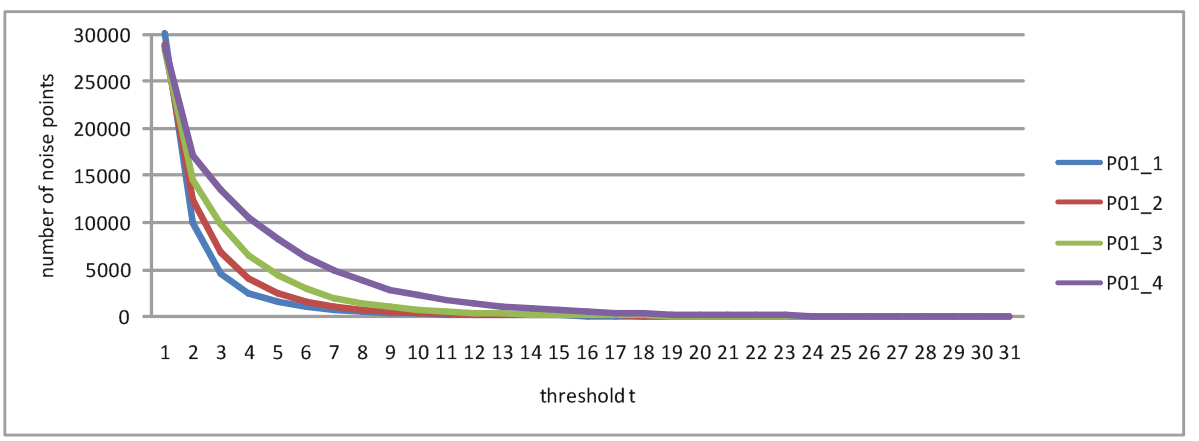

Fig. 3. The obtained curves for one patient P01. Each curve represents one image with different quality. The better quality, the higher maximum curvature. The blue, red, green and violet curves correspond to image (a), (b), (c) and (d) from Fig. 2, respectively. Thus, the blue curve corresponds to the best image, whereas the violet curve corresponds to the worst one. The vertical axis represents $I(t)$, whereas the horizontal one represents the threshold value $t$. (Color figure online)

\section{Results}

In order to verify the described non-reference method, the quality of MR images has been assessed. The calculations were based on images taken for 38 patients. The total number of images was 2708. The number of taken images varied from patient to patient and ranged from 52 to 120 pictures. For each patient, the images were ordered by an expert according to the decreasing quality. The result obtained for one patient is a series of images made with four different exposure times what caused various quality of the images. In addition, imaging was performed on various parts of the body such as the hip joint, spine, knee etc. For a given patient images of only one part of the body were taken, all in the same position. Data used in this paper were described in detail in the article [24].

As it has been noticed in the previous section, the number of pixels, identified as impulse noise for an image, with changing threshold value can be used to assess the quality of a given image. Therefore, each image is represented by a curve that describes the change in the number of peaks isolated with a changing threshold. Next, the function was fitted to each curve and its parameters $a$ and $b$ were estimated. The obtained determination coefficient $R^{2}$ in each case was above 0.95. Based on the estimated parameters of the fitted curves, the maximum curvature $\kappa$ was calculated for each of them. An example of a sequence of values of $\kappa$ for two patients is shown in Fig. 4.

The images were ordered according to decreasing quality. The better the image quality, the greater value of the maximum curvature $\kappa$. The relationship, however, is statistical. The adjusted trend for each $\kappa$ coefficient sequence is a decreasing function. Also, the mean of the curvature coefficients for each of the four groups of images obtained for one patient indicates a decreasing image quality - see Fig. 5. 


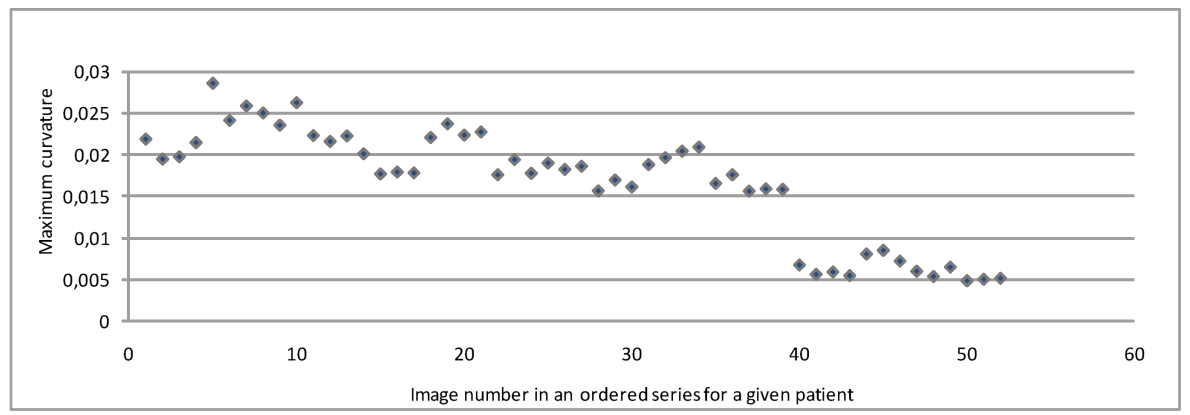

(a)

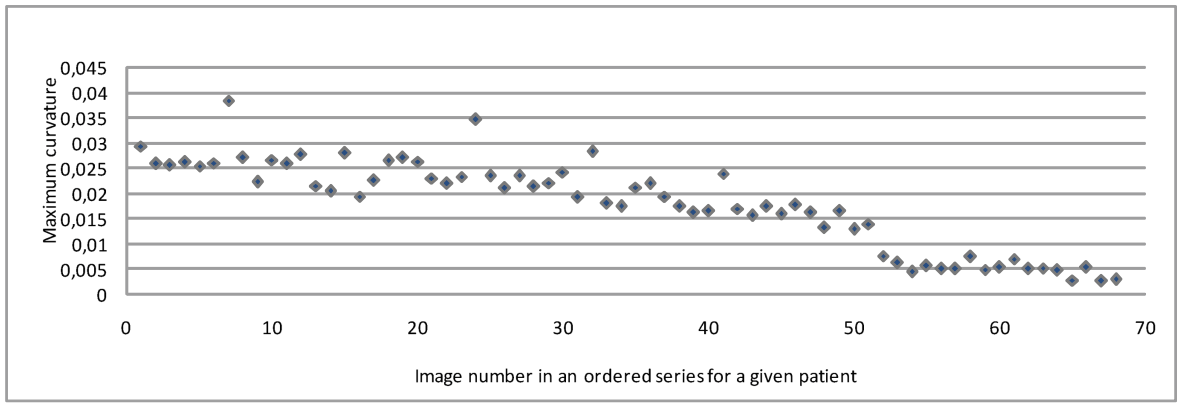

(b)

Fig. 4. Curvature coefficient values for series of images obtained for two patients.

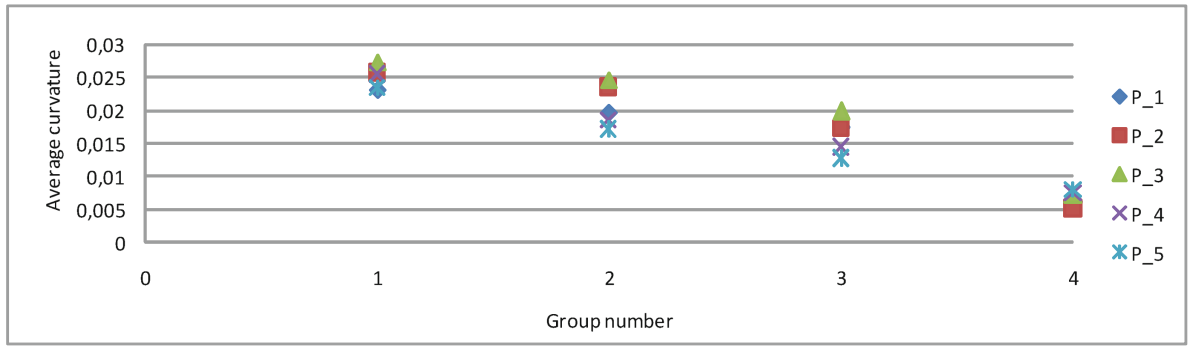

Fig. 5. The mean curvature coefficient values for each of the four highlighted groups for the five selected patients $P_{-} 1, P \_2, P \_3, P \_4, P \_5$

The statistical nature of the relationship between the maximum curvature of the obtained curve and the image quality means that outliers may appear in the neighboring groups. As a result of observation of the analyzed curves, it was noticed that for images of higher quality, the maximum curvature $\kappa$ is higher and occurs for a lower threshold value than for images of lower quality - see Fig. 6 . The correlation ratio between the maximum value of $\kappa$ coefficient for a given patient and the image quality according to the arrangement done by an expert 


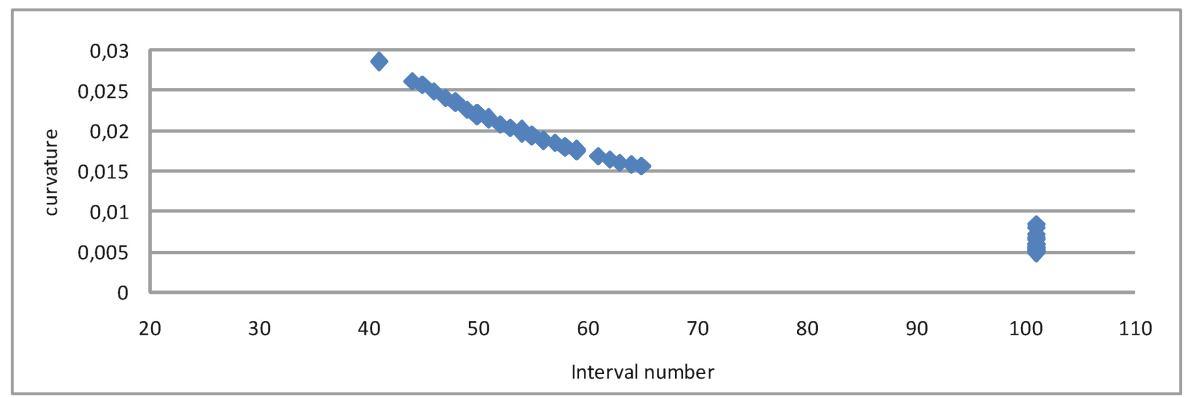

(a)

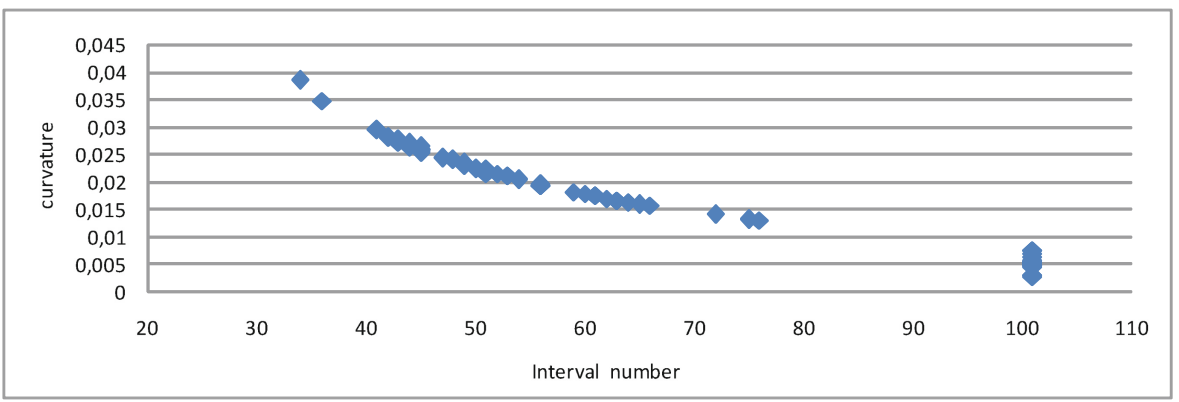

(b)

Fig. 6. Curvature coefficient values depending on the adopted threshold for the patients from Fig. 4

is $r=0.74$. It should be stressed that the correlation is greater than for the method based on entropy, for witch $r=0.67$ and for other methods - see [23].

\section{Concluding Remarks}

The obtained results confirm the strong relationship between image quality and the maximum curvature of the curve obtained on the basis of the quantification of impulse noise in the analyzed image. For an ordered sequence of images due to their quality, the obtained $\kappa$ coefficient values form an ordered descending sequence of values concordant statistically with the sequence of images ordered by an expert. As it has been specified, the correlation ratio is equal to $74 \%$. This means that for higher quality images, the $\kappa$ ratio has, statistically, a higher value for lower threshold values. Thus, the method of automatic assessment of the image quality based on the proposed approach is more effective than the methods described in literature - see [23] and references given there. Therefore, the proposed method is a significant step towards automatic choice of the radiation dose during investigation. Another problem is how the proposed method will evaluate the image after filtering. Among other things, it is planned to be the subject of further studies. 


\section{References}

1. Bielecka, M.: Syntactic-geometric-fuzzy hierarchical classifier of contours with application to analysis of bone contours in X-ray images. Appl. Soft Comput. 69, 368-380 (2018)

2. Bielecka, M., et al.: Modified jakubowski shape transducer for detecting osteophytes and erosions in finger joints. In: Dobnikar, A., Lotrič, U., Šter, B. (eds.) ICANNGA 2011. LNCS, vol. 6594, pp. 147-155. Springer, Heidelberg (2011). https://doi.org/10.1007/978-3-642-20267-4_16

3. Bielecka, M., et al.: Application of shape description methodology to hand radiographs interpretation. In: Bolc, L., Tadeusiewicz, R., Chmielewski, L.J., Wojciechowski, K. (eds.) ICCVG 2010. LNCS, vol. 6374, pp. 11-18. Springer, Heidelberg (2010). https://doi.org/10.1007/978-3-642-15910-7_2

4. Bielecka, M., Korkosz, M.: Generalized shape language application to detection of a specific type of bone erosion in X-ray images. LNAI 9692, 531-540 (2016)

5. Bielecka, M., Obuchowicz, R., Korkosz, M.: The shape language in application to the diagnosis of cervical vertebrae pathology. PLoS ONE 13(10), 17 (2018). Article number e0204546

6. Chandler, D.M.: Seven challenges in image quality assessment: past, present, and future research. ISRN Sig. Process. 7 (2013). Article ID 356291

7. Chow, L.S., Paramesran, R.: Review of medical image quality assessment. Biomed. Signal Process. Control 27, 145-154 (2016)

8. Chow, L.S., Rajagopal, H.: Modified-BRISQUE as no reference image quality assessment for structural MR images. Magn. Reson. Imaging 43, 74-87 (2017)

9. Deshmane, A., Gulani, V., Griswold, M.A., Seiberlich, N.: Parallel MR imaging. J. Magn. Reson. Imaging 36, 55-72 (2012)

10. Dietrich, O., Raya, J.G., Reeder, S.B., Reiser, M.F., Schoenberg, S.O.: Measurement of signal-to-noise ratios in MR images: influence of multichannel coils, parallel imaging, and reconstruction filters. J. Magn. Reson. Imaging 26(2), 375-385 (2007)

11. Elojeimy, S., Tipnis, S., Huda, W.: Relationship between radiographic techniques (kilovolt and milliampere-second) and CTDIVOL. Radiat. Prot. Dosim. 141(1), 43-49 (2010)

12. Flasiński, M.: Introduction to Artificial Intelligence. Springer, Cham (2016). https://doi.org/10.1007/978-3-319-40022-8

13. Flasiński, M.: Syntactic Pattern Recognition. World Scientific, Singapore (2019)

14. Gedamu, E.L., Collins, D., Arnold, D.L.: Automated quality control of brain MR images. J. Magn. Reson. Imaging 28(2), 308-319 (2008)

15. Geissler, A., Gartus, A., Foki, T., Tahamtan, A.R., Beisteiner, R., Barth, M.: Contrast-to-noise ratio (CNR) as a quality parameter in fMRI. J. Magn. Reson. Imaging 25(6), 1263-1270 (2007)

16. Hore, A., Ziou, D.: Image quality metrics: PSNR vs. SSIM. In: 20th International Conference on Pattern Recognition, pp. 2366-2369. IEEE (2010)

17. Huynh-Thu, Q., Ghanbari, M.: Scope of validity of PSNR in image/video quality assessment. Electron. Lett. 44(13), 800-801 (2008)

18. Huda, W., Abrahams, R.B.: Radiographic techniques, contrast, and noise in X-ray imaging. Am. J. Roentgenol. 204(2), 126-131 (2015)

19. Jang, J., Bang, K., Jang, H., Hwang, D.: Alzheimer's disease neuroimaging initiative quality evaluation of no-reference MR images using multidirectional filters and image statistics. Magn. Reson. Med. 80(3), 914-924 (2018) 
20. Ludlow, J.B., Ivanovic, M.: Comparative dosimetry of dental CBCT devices and 64-slice CT for oral and maxillofacial radiology. Oral Surg. Oral Med. Oral Pathol. Oral Radiol. Endod. 106, 106-114 (2008)

21. Mafi, M., Martin, H., Adjouadi, M.: High impulse noise intensity removal in MRI images. In: IEEE Signal Processing in Medicine and Biology Symposium (SPMB), pp. 1-6 (2017)

22. Miao, J., Huang, F., Narayan, S., Wilson, D.L.: A new perceptual difference model for diagnostically relevant quantitative image quality evaluation: a preliminary study. Magn. Reson. Imaging 31, 596-603 (2013)

23. Obuchowicz, R., Oszust, M., Bielecka, M., Bielecki, A., Piórkowski, A.: Magnetic resonance image quality assessment by using non-maximum suppression and entropy analysis. Entropy 22(2) (2020). Article number e22020220

24. Obuchowicz, R., Piórkowski, A., Urbanik, A., Strzelecki, M.: Influence of acquisition time on MR image quality estimated with nonparametric measures based on texture features. Biomed Res. Int. 10 (2019). Article ID 3706581

25. Ogiela, M.: Languages of shape feature description and syntactic methods for recognition of morphological changes of organs in analysis of selected X-ray images. In: Proceedings of Medical Imaging 1998, vol. 3338, pp. 1295-1305 (1998)

26. Ogiela, M., Tadeusiewicz, R.: Syntactic pattern recognition for X-ray diagnosis of pancreatic cancer-algorithms for analysing the morphologic shape of pancreatic ducts for early diagnosis of changes in the pancreas. IEEE Eng. Med. Biol. Mag. 19, 94-105 (2000)

27. Ogiela, M., Tadeusiewicz, R., Ogiela, L.: Image languages in intelligent radiological palm diagnostics. Pattern Recogn. 39, 2157-2165 (2006)

28. Okarma, K., Fastowicz, J.: No-reference quality assessment of 3D prints based on the GLCM analysis. In: 21st International Conference on Methods and Models in Automation and Robotics (MMAR), pp. 788-793. IEEE (2016)

29. Osadebey, M., Pedersen, M., Arnold, D., Wendel-Mitoraj, K.: Bayesian framework inspired no-reference region-of-interest quality measure for brain MRI images. J. Med. Imaging 4(2), 502-504 (2017)

30. Oszust, M.: No-reference image quality assessment using image statistics and robust feature descriptors. IEEE Signal Process. Lett. 11(24), 1656-1660 (2017)

31. Oszust, M.: No-reference image quality assessment with local features and highorder derivatives. J. Vis. Commun. Image Represent. 56, 15-26 (2018)

32. Schulze, D., Heiland, M., Thurmann, H., Adam, G.: Radiation exposure during midfacial imaging using 4- and 16-slice computed tomography, cone beam computed tomography systems and conventional radiography. Dentomaxillofac. Radiol. 33(2), 83-86 (2004)

33. Sinha, N., Ramakrishnan, A.G.: Quality assessment in magnetic resonance images. Crit. Rev. Biomed. Eng. 38(2), 127-141 (2010)

34. Woodard, J.P., Carley-Spencer, M.P.: No-reference image quality metrics for structural MRI. Neuroinformatics 4(3), 243-262 (2006) 